\title{
Ärztliche Versorgung, sanitäre Verhältnisse und Humanversuche im Frauenkonzentrationslager Ravensbrück
}

\author{
Von Hubert Fischer
}

\section{Die Errichtung des Lagers im Jahre 1939}

Das Frauenkonzentrationslager Ravensbrück (FKL Rav) bei Fürstenberg in Mecklenburg entstand Mitte Mai 1939, als die Insassinnen des Frauengefängnisses Lichtenburg in die von Häftlingen des KL Sachsenhausen auf einem freien Gelände errichteten Baracken verlegt worden sind. In den nächsten Monaten kamen zu den 1415 weiblichen Häftlingen 440 Zigeunerinnen aus dem Burgenland sowie 60 im Reich internierte Polinnen.

Die Leitung des Lagers war in 5 Abteilungen gegliedert, und zwar Kommandantur mit Adjutantur, Politische Abteilung, Schutzhaftlager, Verwaltung und Lagerarzt. Dieser war als Leiter der Abteilung V zugleich Standortarzt der SS.

Das Lager konnte in 16 Baracken (Blocks) je 200 Frauen aufnehmen. Der Block 16 diente für neu Eingelieferte 2 bis 3 Wochen als Quarantäne, und ein weiterer Block stand für die Kranken zur Verfügung.

Die Verantwortung für die Ordnung und Disziplin der Häftlinge hatten SS-Aufseherinnen, die von einer aus Häftlingen bestehenden Lagerpolizei unterstützt wurden.

Ende 1939 waren rund 2000 Frauen im Lager, von denen die größte Gruppe die 440 Zigeunerinnen und die 331 Asozialen bildeten, gefolgt von den Politischen (etwa 200, darunter 25 Jüdinnen), 84 Bibelforscherinnen, 50 Berufsverbrecherinnen und 36 wegen Rassenschande Inhaftierten. Die Vorherrschaft der Kriminellen (mit grünem Winkel an der Bekleidung) bedeutete gerade für die neu eingelieferten politischen Gefangenen (mit rotem Winkel) eine sehr große psychische Belastung und verhinderte die Entwicklung einer Schicksalsgemeinschaft, welche das Lagerleben hätte erträglicher machen können.

Die ärztliche Versorgung der Gefangenen lag anfangs in den Händen deutscher Ärztinnen (Frau Dr. Erika Jansen und Frau Dr. Gerda Weyand) und ab Mai 1940 des SS-Obersturmführers Dr. Walter Sonntag. Ihm unterstanden späterhin in der Regel zwei Lagerärzte, der Lagerzahnarzt, einige SS-Krankenschwestern sowie als weiteres Pflegepersonal Häftlinge. 


\section{Die Erweiterung des Lagers in den Jahren 1940 und 1941}

Im Sommer 1940 stieg die Zahl der inhaftierten Frauen auf 3500, so daß die Wohnbaracken mit je 250 Häftlingen belegt werden mußten. Da in den Konzentrationslagern eine Art Selbstverwaltung der Häftlinge bestand, hatten die Kriminellen die meisten erträglichen Arbeitsplätze (z. B. Küche) und Führungsposten (Blockälteste) besetzt und verstanden es, ihre Vorherrschaft insbesondere gegenüber den Politischen auszuspielen. Sie konnten durch Meldung an die SS jede Mißliebige «fertigmachen».

Bei Verstößen gegen die Lagerordnung war die Prügelstrafe nicht erlaubt, fand aber individuell durch das Wachpersonal Anwendung. Offiziell wurde die Prügelstrafe im Dezember dieses Jahres eingeführt und durfte bis zu 25 Schlägen betragen.

Nach Berichten sollen bisher im Lager 47 Frauen im Zellenhaus an Mißhandlungen gestorben, erfroren oder verhungert sein.

Als neuer Standort- und Lagerarzt kam am 18. Februar 1941 vom KL Mauthausen SS-Obersturmführer Dr. Gerhard Schiedlausky, dem nunmehr auch das Männerlager Ravensbrück und das Jugendschutzlager Uckermark unterstanden.

Bezüglich Transporten von Ravensbrück zum Städtischen HufelandKrankenhaus in Berlin-Buch zwischen Mai 1940 und Dezember 1941 antwortete auf eine telegraphische Anfrage des Präsidenten des Kriegsverbrechergerichtes im Curio-Haus in Hamburg am 7.Mai1948 die Krankenhausverwaltung, daß darüber nichts bekanntgeworden sei.

Auf ein Telegramm des Präsidenten des Kriegsverbrechergerichtes am 10. Mai 1948 an das Standesamt Fürstenberg/Mecklenburg bezüglich der monatlich vom Konzentrationslager Ravensbrück zwischen Mai 1940 und Dezember 1941 gemeldeten Todesfälle erfolgte am 1. Mai von FürstenbergHeckl als Antwort:

$\begin{array}{lrlrlr}\text { Mai } 1940 & \text { keine } & \text { Januar } 1941 & 3 & \text { August } & 16 \\ \text { Juni } & 2 & \text { Februar } & 9 & \text { September } & 13 \\ \text { Juli } & 3 & \text { März } & 8 & \text { Oktober } & 30 \\ \text { August3 } & & \text { April } & 8 & \text { November } & 18 \\ \text { September } & 7 & \text { Mai } & 18 & \text { Dezember } & 18 \\ \text { October } & 4 & \text { Juni } & 12 & & \\ \text { November } & 9 & \text { Juli } & 8 & \text { (Insgesamt } & 194 \text { ) } \\ \text { Dezember } & 5 & & & & \end{array}$


Als im Juni die Zahl der Häftlinge auf etwa 6000 angestiegen ist, wurden die hygienischen Verhältnisse im Lager völlig unzureichend. Der Krankenblock war stets überfüllt, da die Schwachen und Kranken immer mehr zunahmen.

Ab 1941 hatte sich die Ernährung von Woche zu Woche verschlechtert. Es gab 500g Brot, $1 / 2$ bis $3 / 4$ Liter Gemüse, einige Kartoffeln sowie Morgenund Abendsuppe, dazu Samstags und Sonntags Wurst oder einen kleinen Käse sowie wöchentlich einen Löffel Schmalz und etwas Marmelade. Fleischgerichte wurden nur am Sonntag ausgegeben.

\section{Weitere Verschlechterung im Jahre 1942}

Anfang des Jahres 1942 gingen mehrere Transporte kranker Frauen (etwa 2150, darunter über 800 Jüdinnen) nach Auschwitz ab. Die Zugänge an Häftlingen umfaßten in steigendem Maße politische Gefangene und Frauen europäischer Widerstandsbewegungen, besonders aus Polen. Die Prügelstrafe wurde im April verschärft, d.h.nach einem Rundschreiben des Reichsführers SS und Chefs der deutschen Polizei, Heinrich Himmler, hatte der Strafvollzug auf das unbekleidete Gesäß zu erfolgen. Im Lager führte sie ein weiblicher krimineller Häftling, der dafür zusätzliche Verpflegung erhielt, mit einem Ochsenziemer aus Leder im Keller des Zellenbaues aus. Beim Strafvollzug mußte stets der Lagerarzt anwesend sein, der auch die Durchführung bestätigte. Für eine körperliche Züchtigung war in jedem Falle unter Beifügen eines lagerärztlichen Gutachtens die persönliche Genehmigung des Reichsführers SS einzuholen.

\section{Die Humanversuche an Häftlingen}

Im Juli 1942 begann eine klinische Versuchsreihe über Gasbrand an jungen Polinnen, die als Angehörige der polnischen Intelligenz und der Widerstandsbewegung verhaftet und in der Mehrzahl zum Tode verurteilt waren. 13 Polinnen sind bereits am 18. April hingerichtet worden. Aus Platzgründen wird auf ihre Darstellung verzichtet. 


\section{Vergrößerung des Lagers und Einsatz der Frauen in der Rüstungsindustrie ab 1942}

Im Sommer erfolgte eine Vergrößerung des Lagers. Hinter der Lagermauer entstanden große stabile Gebäude mit Arbeitsplätzen für mehrere Tausend Häftlinge und auf der anderern Seite Baracken der Firma Siemens-Halske. Die Aufstellung neuer großer Wohnblocks diente zur Unterbringung weiterer Frauen. Alle diese Bauten wurden von Häftlingen des benachbarten Männerlagers ausgeführt. Schließlich standen im Frauenlager 32 Wohnbaracken an 3 Lagerstraßen. Wegen der Überbelegung lagen die Frauen zu dritt auf 2 Strohsäcken, saßen zu zweit auf einem Schemel und teilten sich zu viert in einen Kasernenspind. Wäsche, Bekleidung und Unterbringung wurden immer schlechter, ebenso die Verpflegung.

Ordnung und Disziplin lockerten sich in einer alle bedrohenden Weise. Das Auftreten von Kleiderläusen, offenbar durch russische Zugänge, veranlaßte die Lagerleitung zu rigorosen Desinfektionsmaßnahmen. Nach Wechsel des Lagerkommandanten erfolgte die Verhaftung des Lagerarztes SSUntersturmführer Dr. Ralph Rosenthal und des Häftlings Gerda Quernheim, einer ehemaligen Krankenschwester. Wie Frau Buber-Neumann schrieb, wurde «Rosenthal vor ein SS-Gericht gestellt und zu sieben Jahren Zuchthaus verurteilt, Gerda Quernheim von Ramdor (neuer Schutzhaftführer) in den Zellenbau gesperrt und später nach Auschwitz abgeschoben. Der Verbrechertätigkeit dieser beiden setzte die Aussage eines tschechischen Häftlingsarztes aus dem Männerlager Ravensbrück ein Ende. Gerda Quernheim war zweimal schwanger gewesen, und zur Abtreibung rief Rosenthal den Häftlingsarzt zu Hilfe, der dann die Verhaftung des Paares veranlaßte. Die Morde an Frauen und Neugeborenen hätten den SS-Arzt nie gestürzt, aber man wußte von systematischen Diebstählen der Goldzähne seiner Opfer zu berichten.»

Rosenthal hat später zugegeben, 20 bis 30 Kranken eine Überdosis von Morphium verabreicht zu haben.

«Die neuen SS-Ärzte begannen in Ravensbrück tschechische, deutsche, russische und polnische Häftlingsärztinnen zur Behandlung der kranken Gefangenen heranzuziehen. Dadurch verlor das Revier viele seiner Schrecken, wenn auch manche Häftlingsärztinnen sich in Ton und Gebaren bald wenig von ihren SS-Kollegen unterschieden. Zudem gab es auch solche, die die Kranken ihrer Nation bevorzugt behandelten» (BuberNeumann). 
Bekannt sind die Namen folgender Ärztinnen, welche nach dem Krieg als Zeuginnen aussagten: Dr. Louise Le Porz (Französin), Zdenka Hedvedova (Tschechin), Wisia Zurkowska, Dr. Helene Goudsmith (Belgierin), Dr. Denise Fresnel (Französin), Dr.Zofia Haczka, Dr. Doris Maase (April 39 bis Juli 41), Dr. Anni Rudroff.

Der Kriminalassistent Ludwig Daniel Ramdor ließ bei seinen brutalen Verhörmethoden durch den Häftlingsarzt vom Männerlager Narkotika injizieren.

Bereits im Sommer war eine Massenpsychose unter dem Bild der spinalen Kinderlähmung aufgetreten. Die Belegstärke des Lagers betrug nunmehr etwa 10000 Frauen.

Ab November konnten den Häftlingen Pakete mit Lebensmitteln und Kleidung in unbeschränktem Umfang geschickt werden. Die Häftlinge des Auslandes wurden mit guten Lebensmitteln versorgt, wie Finkelmeier vom Männerlager berichtete.

Bezüglich des Meldeverfahrens bei Todesfällen in Konzentrationslagern verfügte der Amtsgruppenchef D im SS-Wirtschaft-Verwaltungshauptamt am 21. November 1942, daß Todesfälle von Juden und Jüdinnen nur noch in einer Sammelliste zu erfassen, Todesmeldungen über alle anderen Häftlinge mit dem bisher verwendeten Formblatt einzureichen sind. Dabei ist es gleichgültig, ob natürliche oder unnatürliche Todesfälle vorliegen. Als letzter Satz stand: «Die Lagerkommandanten sind dem Reichsführer SS und mir persönlich dafür verantwortlich, daß trotz dieser Verminderung der Meldung an keiner Stelle in den Lagern vergessen wird, dass auch das Menschenleben eines jeden Verbrechers verantwortet werden muß.»

Am 2. Dezember wies der Reichsführer SS darauf hin, daß die Prügelstrafe keine Erziehungsmaßnahme darstelle, und ordnete ihre Anwendung nur als letztes Mittel an. Sie durfte künftighin erst nach Ausschöpfung aller Disziplinarmaßnahmen beantragt werden.

1942/43 betrug die durchschnittliche Belegstärke des Lagers 10000 bis 15000 Frauen bei 400 bis 500 Todesfällen. Neben dem Hauptlager bestanden zahlreiche Außenkommandos auch in Bayern, Sachsen und im Sudetenland, insgesamt 33 mit sehr unterschiedlicher Belegungsstärke.

Die ärztliche Aufnahmeuntersuchungen von neu eingelieferten Frauen schienen oberflächlich und entwürdigend gewesen zu sein, wie Augenzeuginnen berichteten. 


\section{Eintreffen weiblicher sowjetrussischer Kriegsgefangener}

Ende Februar 1943 kamen 536 weibliche sowjetrussische Kriegsgefangene (meist von der Krim, Sewastopol) in das Lager, darunter viele Ärztinnen und Sanitätspersonal. Sie blieben 4 Wochen in Quarantäne und wurden dann im Roten-Armee-Block untergebracht.

\section{Das Jahr 1943}

Die Überfüllung des Lagers hielt an, wenngleich zahlreiche Transporte in die Außenkommandos und andere Lager abgingen. Andererseits kamen aus diesen Lagern kranke und nicht mehr arbeitsfähige Frauen zurück.

Anfang Mai erschien im Befehlsblatt des Chefs der Sicherheitspolizei und des SD unter Nummer 23/1943 ein Verbot der Einweisung schwangerer Häftlinge in das Frauenkonzentrationslager Ravensbrück bzw.in die Frauenabteilung der Konzentrationslager Auschwitz und Lublin. «Wird eine Schwangerschaft ärztlicherseits festgestellt, ist von der Überführung in jedem Falle abzusehen», hieß es. Bis zum Frühjahr kamen die Leichen Verstorbener zur Einäscherung in das städtische Krematorium.

Eine Zustimmung zur Sterilisation schwachsinniger Frauen hat Dr. Schiedlausky niemals gegeben, da Ravensbrück kein für diese Operationen autorisiertes Krankenhaus war (Aussage JAG 225).

Nach Dr. Schiedlausky, der im August von Dr. Richard Trommer (bis April 1945) abgelöst wurde, sind von Mitte Dezember 1941 bis August 1943 etwa 44 Frauen für die Konzentrationslager-Bordelle ausgesucht worden, die Polinnen zumeist für Bordelle der ukrainischen Wachmänner. Nach der Verfügung des Amtsgruppenchefs D vom 20. November mußten die Aufseherinnen in den Häftlingssonderbauten (Bordellen) bis 10.12.43 zurückgezogen werden. An ihre Stelle traten ältere weibliche Häftlinge. «Beim FKL Ravensbrück stehen erfahrene weibliche Häftlinge zur Verfügung, die bereits Bordelle geleitet haben.» Insgesamt sollen nach Mauthausen 16 Dirnen verlegt worden sein, nach Buchenwald 14, nach Flossenbuerg 10 und nach Dachau 2 bis 4 . Im Lager Ravensbrück haben sich auch lesbische Beziehungen entwickelt. Im August betrug die durchschnittliche Belegstärke des Lagers 13100 bei 38 Todesfällen.

Im August erfolgte auf Befehl des Reichsführers SS eine Trennung der politischen Häftlinge von den kriminellen. 
Gegen Ende 1943 ging die Sterblichkeit im Lager trotz zunehmender Belegung monatlich auf 0,2\% zurück. Eine Statistik für 1943 bis 1945 soll bei den Papieren von Dr. Treite liegen. Danach stieg die Sterblichkeit bis April 45 auf monatlich 5\%. Dies beruhte darauf, daß alle kranken weiblichen Häftlinge aus den KL Deutschlands nach Ravensbrück gebracht wurden, die Belegung im Lager auf etwa 35000 Frauen stieg und auf dem langen Aufenthalt der unterernährten und völlig unzureichend bekleideten Häftlinge im Freien während des Winters.

\section{Zunahme der Todesfälle infolge Epidemien, unzureichender Ernährung und Sklavenarbeit im Jahre 1944}

Zu Beginn des Jahres 1944 wurden die Disziplinarstrafen gegen die Aufseherinnen in Konzentrationslager geregelt.

Über den Sanitätsdienst des «Industriehofes», den eine junge tschechische Medizinstudentin versah, berichtete Buber-Naumann: «Ich stand in langer Schlange mit den anderen Kranken im engen Gang zwischen den Kojenreihen, wo Inka auf einem Holzschemel ihren Verbandsstoff, ihre Salben und Desinfektionsmittel ausgebreitet hatte und nun eine nach der andern verarztete. Sie war zu bewundern in ihrem unermüdlichen Eifer und der nie versagenden Freundlichkeit.»

Im Lager wurden Schwerkranke ohne Heilungschancen mit Spritzen getötet. Die Lagerärztin, Fräulein Dr. Oberhäuser, gestand, 5 bis 6 Frauen in die Armvene $10 \mathrm{~cm}^{3}$ Petroleum injiziert zu haben. Sie bäumten sich nach 3-5 Minuten auf und brachen dann zusammen. Nach Aussage der angeklagten Ärztin hatte es sich um Schwerkranke gehandelt, die nicht mehr geheilt werden konnten (NOKW/d 601-651).

Das große Sterben in Europa erfaßte auch in besonders brutaler Weise die Konzentrationslager. In Ravensbrück diente das Krankenrevier I für Chirurgie und allgemeine Krankheiten, während das Krankenrevier II die Infektionsabteilung bildete.

Eine ursprünglich für 260 Frauen vorgesehene Baracke beherbergte bis zu 800. Die großen Baracken faßten bis zu 1400 Frauen. Die zahlenmäßig zu wenig Toiletten lagen viel zu weit entfernt.

Mit den sich ausbreitenden Epidemien mußte ein Block nach dem anderen für das Revier beschlagnahmt werden. Sieben bis acht Blocks wurden Krankenblocks bzw. «Bettkartenblocks», d.h. Blocks, die leichtere 
Kranke, welche vom «Appellstehen» dispensiert waren, für einige Tage beherbergten. So lagen 500 Frauen im Block 13 in Quarantäne. Durch immer wieder neu auftretende Scharlacherkrankungen mußte die Quarantäne insgesamt 5 Monate aufrechterhalten werden.

«Es gab auch eine spezielle Entlausungskolonne, die von Zeit zu Zeit kam, sämtliche Insassen des Blocks am frühen Morgen hinauswarf, Strohsäcke, Decken und Bündel mit einer stinkenden Flüssigkeit übergoß und alles derart durcheinander warf, daß es einem selten gelang, später zu seiner eigenen Decke zu kommen. In der gleichen Zeit warteten wir einen ganzen Tag stehend, entweder im Duschraum oder später, als dieser durch die zahlreichen Neuankömmlinge ständig belegt und am Ende in die Gaskammer umgewandelt war, in einem riesigen, bitterkalten, zugigen Zelt auf das Ende der Entlausung. Wir mußten uns dann entkleiden, und in großem Gewühl und sehr summarisch suchte man die behaarten Stellen unseres Körpers mit einer Zahnbürste nach allerlei Arten von Läusen - es gibt deren viele Spezies - ab, bestreute unsere Kleider mit einem ziemlich unwirksamen Pulver und entließ uns in den Block. Dort fanden wir außer einem heillosen Durcheinander unsere Betten noch völlig feucht und verwüstet vor.

Abgesehen von der Aussicht, bei dieser Prozedur Rheumatismus oder eine schlimme Erkältung zu erwischen, hatten wir nichts gewonnen - denn die Läuse triumphierten weiter, wenn sie auch, fliehend von einem Körper zum anderen, den Besitzer wechselten»(Wagner).

Wenngleich die Anständigkeit des SS-Apothekers (SS-Hauptscharführer Lorbeer) von den Häftlingen erwähnt wird, so wußte man doch, dass von den SS-Ärzten keine Medikamente erhältlich waren. Es wurden Kohletabletten selbst hergestellt und von den Außenkolonnen Schafgarbe, Kamille, Pfefferminze, Birkenblätter und Maiwuchs von Nadelbäumen mitgebracht.

Die SS-Oberschwester Marschall hatte als Helferinnen Häftlingsschwestern. Weiter werden die SS-Schwestern Margarethe Hoffmann und Erika Milleville genannt (Buchmann). Der 2. Lagerarzt, Dr. Percy Treite, suchte 800 Frauen für einen Transport nach Lublin aus.

Nach Frau Vermehren wurden im Frühjahr im Keller des Zellenbaues Erschießungen mit schallgedämpfter Pistole vorgenommen, darunter 21 Polinnen. Zudem berichtete Frau Vermehren, daß die 8 Klosetts im Block II für seine vierhundertköpfige Besatzung nicht ausreichten. Es herrschte damals auch eine anhaltende Typhusepidemie, die zeitweise täglich bis zu 70 Opfer forderte. 


\section{Die Belegung des Lagers 1944}

Die Belegung des Lagers verteilte sich damals folgendermaßen:

Block I Küchenblock. Hier waren auch 250 in der Küche arbeitende Polinnen untergebracht, ebenso Schreibstubenpersonal und andere avancierte Häftlinge.

Block II Unterkunft der in der Verwaltung tätigen Frauen.

Block III ähnliche Personengruppen. Nur wenige Frauen mußten ihr Bett mit einer anderen teilen.

Block IV Unterbringung der doppelten Häftlingszahl bei gleich vielen Betten, dadurch Überbelegung.

Block V Bibelforscherinnen sowie Masse der neu Eingeglieferten: 1300 Frauen auf 450 Betten.

Block VI-VIII waren dem Revier zugeteilt.

Block IX-XXXI wurden als Slums bezeichnet wegen der hier herrschenden entsetzlichen Zustände.

«Die ärztliche Untersuchung der Arbeitsfähigen beschränkte sich auf die Ausscheidung der Humpelnden, der Buckligen und der Krätzekranken unsichtbare Leiden wurden nicht berücksichtigt» (Vermehren).

Die zahlreichen Geisteskranken sind schließlich im Dienstzimmer eines Revierblockes eingesperrt worden, insgesamt 69. Die Irren wurden 2mal täglich zum Austreten herausgelassen, wenngleich es meist schon im Zimmer besorgt worden war. Dreimal täglich erhielten sie eine halbe EBportion. Damals diente Block 22 als Quarantäne-Aufenthalt. Die Abstellung von Häftlingen für Versuchszwecke war in jedem Falle von einer Genehmigung des Amtsgruppenchefs D abhängig, worauf in einer Verfügung vom 12.Mai 1944 nochmals hingewiesen wurde.

\section{Neue Zugänge aus dem Osten}

Als im September/Oktober 1944 etwa 12000 Polinnen aus Warschau eingeliefert wurden, erreichte das Elend im Lager seinen Höhepunkt. Die Blockälteste sammelte jeden Morgen die Kranken für das Revier. Frau Wagner brachte sie dann dorthin. Außer einem SS-Arzt gab es nur Häftlingsärztinnen. Im Vorraum amtierte eine sogenannte Schwester, das Böseste, das man sich denken konnte, wie in Erinnerungen zu lesen ist. 
Schrieb die Ärztin eine Kranke für den Revierblock auf, mußte sie nicht mehr am täglichen Appell teilnehmen (etwa 2 Stunden stehen bei jeder Witterung).

Jeder Block bestand aus einem Tages- und einem Schlafraum für ursprünglich 240 Häftlinge. Nunmehr hausten allein auf einer Seite 530 Frauen, insgesamt an die 1100. Jeder Strohsack in den dreistöckigen Bettgestellen war doppelt und dreifach belegt. Es herrschte eine Läuseplage, und das Wasser durfte wegen Typhusgefahr nicht getrunken werden. Nach dem Wecken um 4 Uhr standen am Klosett 500 Frauen an 3 Schüsseln an, vor denen es keine Türen gab. Zwei Schüsseln konnten nicht benutzt werden. In den Räumen wurde alles gestohlen, besonders Schuhe und Kleidung. In einem Block waren hauptsächlich Französinnen und Polinnen untergebracht. Die Norwegerinnen zeigten sich sehr kameradschaftlich.

Ende November kamen mit einem Transport aus Auschwitz viele ungarische Jüdinnen ins Lager. Die insgesamt 3000 Frauen waren in zwei Wochen $300 \mathrm{~km}$ zu Fuß marschiert, wobei durch strengen Frost viele Erfrierungen aufgetreten sind. Das Lager Auschwitz war vor den heranrükkenden sowjetrussischen Truppen geräumt und die Belegschaft nach Westen getrieben worden. "Als die Tausende von Frauen Ravensbrück erreichten, waren ihre Gesichter vor Hunger und Durst vertiert. Sie schrien und bettelten um Wasser. Tagelang hatten sie keinen Tropfen erhalten. Die Küchenhäftlinge baten um die Erlaubnis, den Dürstenden Kaffee bringen zu dürfen. Es geschah, aber Hunderte stürzten sich über die Kübel, schlugen sich gegenseitig zu Boden und der Kaffee floß auf die Erde. Erst die prügelnde Lagerpolizei und ein Heer von Aufseherinnen machten es möglich, das Getränk an die Verdurstenden zu verteilen. Die Auschwitzer Häftlinge brachten ihren eigenen Lebensstil mit. Den hatte das Vernichtungslager geprägt: Koste es was es wolle, aber rette dein Leben! Sie waren robust und brutal in Aussehen und Manieren» (Buber-Neumann). Nach Aussage von Dr. Treite, der im Herbst nach Ravensbrück versetzt wurde, führte dieser Transport zum stärksten Anfall an Toten und Kranken. Von den körperlich und seelisch völlig heruntergekommenen Überlebenden starben noch etwa $50 \%$.

Von Auschwitz stammte auch Dr. Winkelmann, der nun kranke und nicht arbeitsfähige Frauen für das Jugendlager Uckermark ausmusterte, einem Lager «Mittweida». Von dort kamen viele in das lagereigene, Ende April 1943 gebaute Krematorium, welches in diesem Jahr einen zweiten Ofen erhalten hatte. Dr. Winkelmann war der Nachfolger von Dr. Lukas, der 
sich geweigert hatte, an den Aussonderungen der Kranken (zu alt, zu mager, Beinödem) teilzunehmen.

Im Dezember erfolgte die Inbetriebnahme einer Gaskammer mit einem Fassungsvermögen von etwa 150 Personen. In diesem Jahr betrugen die Todesfälle 3,0 bis $3,5 \%$.

Dr. Treite sagte später vor Gericht aus, daß er auf einen Befehl, geistesschwache Frauen deutscher Nationalität zu sterilisieren, selbst 10 bis 15 Sterilisationen ausgeführt habe. «Technisch wurde entweder eine Exstirpation der Eileiter (Tube) oder eine Exzision aus dem mittleren Teil der Eileiter ausgeführt. Komplikationen traten niemals ein. Professor Clauberg führte Sterilisationen von Zigeunerinnen im Röntgenzimmer des Reviers aus. Ich hörte, daß es sich um Injektionen gehandelt hat.»

\section{Der schreckliche Winter 1944/45}

An Weihnachten 1944 befanden sich in Ravensbrück über 400 Kinder, für die von den gefangenen Frauen eine kleine Bescherung veranstaltet worden ist.

Nach Don Zimmet starben in Ravensbrück 1944/45 von den nicht provozierten Todesfällen an:

Dysenterie (Ruhr) etwa $50 \%$

Hungerödem, Kachexie (Abzehrung)

Tuberkulose

Pneumonie (Lungenentzündung)

Typhus, Paratyphus

akuter Nephritis (Nierenentzündung) 5

Herzerkrankungen 3

Furunkulose, Abszessen, Septikämie (Blutvergiftung) 3

Fleckfieber 2

Otitis, Mastoiditis (Mittelohrenentzündung) 1

anderen Infektionskrankheiten 1

In diesem Winter lagen die Toten im Leichenkeller bis zu ihrer Verbrennung. Dazu Buchmann: «Mitten zwischen ihnen hantiert eine russische Ärztin mit den scharfen Messern. Auf Befehl seziert sie unermüdlich, blaß und schmal, Bitterkeit in dem übermüdeten Gesicht. Wozu, warum sezieren?»

«Und den Toten bricht man die «Goldzähne aus - Zahnarzt Dr. Hellinger betreibt das schauerliche Handwerk persönlich.» 
Im Block 10 lagen fast 500 Tuberkulosekranke, 200 in einem Raum. Zeitweise gab es für die 500 Kranken nur einen Fieberthermometer. Hier

arbeiteten zwei französische Häftlingskrankenschwestern sowie die Ärztin Louise Le Porz aus Bordeaux. Weiterhin erwähnte Buchmann anerkennend die Tätigkeit der Ärztinnen Dr. Mlada Tauferova (Röntgen) und Dr. Donna Naliwaiko.

An den im Winter 1944/45 am späten Abend vor dem Krematorium des Lagers stattfindenden Erschießungen hatte Dr. Treite teilzunehmen zur Feststellung des Todes.

\section{Das Massensterben bis zur Befreiung}

Im Januar 1945 waren etwa 21000 politische Gefangene im Lager, 3000 Berufsverbrecherinnen sowie kleine Gruppen von Bibelforschern, sexuell Abnormen, Zigeunerinnen und Asozialen. Sie hausten in 16 Baracken für je 250 Frauen und großen Baracken für je 450 Frauen. Die Belegung erreichte 17500 und sogar über 25000 . Auf einem Bett mußten 2 bis 3 Frauen mit einem Strohsack und je 2 Decken schlafen. Unter diesen etwa 25000 Frauen befanden sich 10000 bis 11000 Russinnen und 6000 Polinnen. In den beiden ersten Monaten kamen etwa 1000 Häftlinge in das Lager, darunter auch solche von Auschwitz. Auf diesem Transport verstarben von 6000 Frauen an die 2000. Das Revier im inzwischen aufgelösten Jugendschutzlager Uckermark war nur notdürftig eingerichtet und hatte keine Heizung. Die Behandlung führten zwei Häftlingsärztinnen und 4 Häftlingspflegerinnen durch. Anfang Februar lagen 150 bis 180 Patientinnen im Revier, und täglich starben an die 50 Frauen. Die Häftlingsschwester Vera Salvequart gab ein Pulver aus, ein «Schlafmittel», ebenso wie die SS-Schwester Martha im Block 10 auf Anweisung von Dr. Trommers an 50 Schwerkranke. Am nächsten Morgen waren davon 5 tot, dann starben 7 und am nächsten Tag weitere 12.

Im Februar waren 5 Blocks mit Kranken überfüllt, und es begannen Vergasungen. Bereits bis zum 15. sind etwa 4000 Frauen in Ravensbrück vergast worden. Fünf Blocks waren mit Kranken überfüllt. Am 9. Februar erfolgte die Vergasung von 72 tuberkulosekranken Frauen. Andere Kranke erhielten ein weißes Pulver, worauf sie in tiefen Schlaf fielen und dann starben. In den letzten Monaten gab es viele Todesfälle an Bauchtyphus und Fleckfieber sowie Dysenterie. In den Krankenblocks wurden alle 2 bis 3 Wochen Aussonderungen für das Krematorium vorgenommen. 
Vom 14. Februar stammt eine Liste mit 79 Namen von Frauen, die vom Block 10/IV ins Jugendlager überstellt wurden. Ihre Häftlingsnummern reichten von 9414 bis 102773 .

Vom 27.1. bis 23. April gingen nach Buchmann mindestens 297 Frauen vom Block 10 in den gewaltsamen Tod.

In das Anfang Februar 1945 bezogene ehemalige Jugendlager (Baubeginn April 1942) wurden alle alten und arbeitsunfähigen Frauen verlegt sowie ein großer Teil des Transportes aus Auschwitz. Obgleich die Wohnbaracken in gutem Zustand waren, so fehlten ausreichende sanitäre Einrichtungen. Mitte März lagen dort über 5000 Frauen. Ende Februar erhielt der Lagerkommandant einen Befehl vom Reichsführer SS, wonach alle kranken und marschunfähigen Frauen zu töten seien.

«In den darauffolgenden Tagen wurden in den verschiedenen Blocks Selektionen von Dr. Trommer durchgeführt, der dabei über 2300 Frauen heraussuchte. Zuerst begann man mit Erschießen dieser Frauen. Die Erschießungen wurden von SS-Hauptscharführer Moll durchgeführt. Er hatte 8 Häftlinge zu Hilfe. Diese Methode schien dem Lagerkommandanten nicht schnell genug zu sein. Er sagte in meiner Anwesenheit: «Es geht nicht schnell genug, wir müssen andere Methoden anwenden.s Daraufhin ordnete Sturmbannführer Sauer den Einbau einer Gaskammer in einer Baracke neben dem Krematorium an. Ich war bei einem Gasen anwesend. Es wurden immer 150 Frauen auf einmal in die Gaskammer gezwungen. Hauptscharführer Moll gab den Frauen den Befehl sich auszukleiden und teilte ihnen mit, daß sie eine Entlausung mitmachen würden. Daraufhin wurden sie in den Gasraum eingeführt und die Tür verschlossen. Ein männlicher Häftling mit einer Gasmaske versehen kletterte auf das Dach und warf von oben durch eine Öffnung, die er sofort wieder zumachte, eine Gas-Büchse in den Raum. Ich hörte Stöhnen und Wimmern im Raum. Nach zwei bis drei Minuten wurde es still im Raum» (Aussage von SS-Obersturmführer Schwarzhuber).

Ergänzend dazu bemerkte Schwarzhuber, daß in Ravensbrück zwischen 2300 und 2400 Menschen vergast wurden. Die Gaskammer war ungefähr $9 \times 4,5$ Meter und faßte ungefähr 150 Menschen. Sie lag an die fünf Meter vom Krematorium entfernt. Die Frauen mußten sich in einem kleinen Schuppen ausziehen, der drei Meter von der Gaskammer entfernt lag und wurden dann durch ein kleines Zimmer in den Gasraum gebracht. Nach Aussage des SS-Obersturmführers Schwarzhuber starben von Januar bis Mitte Februar täglich durchschnittlich 30 bis 35 Frauen. Mitte Februar bis 
Mitte März erreichte die Sterblichkeit 60 bis 70 täglich, um dann wieder auf 30 bis 35 zurückzugehen. Der Tod erfolgte an epidemisch ausgebrochenen Krankheiten sowie an Körperschwäche infolge der schlechten Verhältnisse im Lager.

Die Zahl der Toten betrug Ende März etwa 6400, nachdem es allein in diesem Monat 1953 Tote gegeben hat. Eine Bestätigung der genannten Zahlen ist nicht möglich, da nach Auskunft der Nationalen Mahn- und Gedenkstätte Ravensbrück vom 4. April 1986 auf dem Gelände des Konzentrationslagers Ravensbrück im April 1945 durch die SS der größte Teil der Registraturen vernichtet wurde.

«Zahlreiche Zeitzeugen berichten, daß sie noch als Häftlinge des $\mathrm{KZ}$ Ravensbrück unter SS-Bewachung, Papiere, Häftlingsakten etc. in großen Mengen im Krematorium des Lagers verbrennen mußten. Die Dokumentensammlung im heutigen Archiv der Gedenkstätte Ravensbrück bietet keine Möglichkeit, beispielsweise exakt das interne Problem der Organisation der Konzentrationslagerführung nachzuvollziehen.»

Auch die städtische Friedhofsverwaltung in Fürstenberg besitzt keine Unterlagen über das Bestattungswesen im KL Ravensbrück.

In der Gaskammer sollen bis zur Befreiung 5793 Frauen vergast worden sein. Aus den letzten Monaten des Lagers berichtete Frau Schaeder, daß die Polinnen es verstanden hätten, sich in großem Ausmaß der Posten auf den Krankenrevieren zu bemächtigen. Eine ältere polnische Ärztin hat dort die Genesung von Frau Schaeder nach schwerer Krankheit mit rührender Sorgfalt gefördert und segensreich auf die Frauen im Krankensaal gewirkt, den der deutsche SS-Arzt nicht betreten hat.

Zwischen 1943 und 1945 kamen im Lager etwa 870 Kinder auf die Welt. Fast alle Säuglinge sind gestorben. "Im Krankenrevier amtierte eine tüchtige Hebamme, und als die Geburten immer zahlreicher wurden, richtete man sogar eine Baracke für die Schwangeren und Neugeborenen ein. Es gab Tage, an denen fünf Kinder das Licht der Welt erblickten. Eine junge Tschechin, Eliska, meldete sich freiwillig zur Arbeit in der Säuglingsbaracke. Sie sammelte Stoffetzen für Windeln und Hemdchen. Vom Lager bekamen die Säuglinge keine Milch, und die unterernährten Mütter konnten ihre Kinder nicht stillen. Man mußte sie mit Zutaten aus den Rot-KreuzPaketen notdürftig ernähren.» In den Kriegsjahren befanden sich bis zu 400 ältere Kinder im Lager.

Im März und Anfang April des Jahres 1945 wurden 21 Frauen hingerichtet (18 Russinnen und 3 Engländerinnen). Etwa im gleichen Zeitraum sind 
durch Intervention des Dänischen und Schwedischen Roten Kreuzes 300 Französinnen, 100 Skandinavierinnen und 100 kranke Frauen entlassen worden. Auf Veranlassung von Prof. Dr. Gebhardt wurden auch die Versuchspersonen dem Schwedischen Roten Kreuz übergeben. Weitere Entlassungen betrafen 2500 Häftlinge.

Damals besuchte ein Delegierter des Internationalen Comité vom Roten Kreuz das Lager und berichtete davon (Comité S. 105/111). Trotz zahlreicher Schwierigkeiten brachten Lastkraftwagen des Internationalen Comité am 5. April 299 Französinnen und eine Polin nach der Schweiz.

Die Situation Mitte April schilderte Frau Schmidt-Fels: «In den letzten Tagen vor der Evakuierung des Lagers gab es kein Brot mehr, nur einen halben Liter Wassersuppe, in der einige schmutzige Kartoffelschalen und gedörrte Steckrüben schwammen; und auch auf diese Suppe mußten wir bis vier Uhr nachmittags warten und dennoch arbeiten, mit leerem Magen. Viele wühlten in den Müllkästen des Elite-Blocks nach weggeworfenen Kartoffelschalen. Das verschimmelte Brot, das dann und wann in einem Paket ankam, war für uns eine Delikatesse. Ich habe Frauen gesehen, die sich mit tierischer Gier auf die Speisereste von Typhus- und Diphtheriekranken warfen, ungeachtet der Ansteckungsgefahr, der sie in der Folge auch kaum entgingen.»

Die letzte Registernummer für Häftlinge war 105000. 1939-45 kamen etwa 132000 Frauen nach Ravensbrück, darunter etwa 40000 Polinnen. Die durchschnittliche Belegungsstärke in den einzelnen Jahren soll betragen haben:

$\begin{array}{rrr}1939 & 2280 & \\ 1940 & 4000 & \\ 1941 & 7900 & \\ 1942 & 10800 & \\ 1943 & 15000 & \\ 1944 & 43000 & \\ 1945 & 50000 & \text { (Kühnrich) }\end{array}$

Bei der Auflösung des Lagers am 29. April 45 befanden sich etwa 12000 weibliche Häftlinge im Lager, darunter 3000 Französinnen, Belgierinnnen und Luxemburgerinnen, 500 Jüdinnen und 4000 Deutsche, sowie 12 Engländerinnen und 14 Amerikanerinnen, der Rest Frauen aus den Balkanstaaten.

Am 27. April begannen die Evakuierungsmärsche, und am 30. April befreite die 49. Abteilung der Sowjetarmee die im Lager Zurückgebliebenen. 
Es waren 2000 bis 3000 kranke und gehunfähige Frauen mit freiwilligem Häftlingspflege-Personal, darunter Frau Vaillant-Couturier.

Nach dem Kriege wurden von alliierten Militärgerichten wegen Mißhandlung und Tötung alliierter Staatsangehöriger im KL Ravensbrück sowie Selektion zur Tötung durch Vergasung von 3000 weiblichen alliierten Staatsangehörigen im Jugendschutzlager Uckermark

zum Tode durch den Strang verurteilt 21 Männer und Frauen, darunter 7 Ärzte,

zu lebenslänglich 1 Mann und eine Frau, darunter ein Arzt, zu 20 Jahren ein Mann und zwei Frauen, darunter eine Ärztin,

zu 15 Jahren drei Männer, darunter ein Zahnarzt,

zu 12 Jahren eine Frau,

zu 10 Jahren ein Mann und vier Frauen,

zu Jahren eine Frau,

zu 3 Jahren eine Frau,

zu 2 Jahren ein Mann.

\section{Literatur}

Antoni, E.: KZ - Von Dachau bis Auschwitz. Faschistische Konzentrationslager 1933-1945. Röderberg, Frankfurt am Main 1979.

Arndt, I.: Das Frauenkonzentrationslager Ravensbrück. In: Studien zur Geschichte der Konzentrationslager. Schriftenreihe der Vierteljahrshefte für Zeitgeschichte; Nummer 21. Deutsche Verlagsanstalt, Stuttgart 1970. S.93-129.

Audoul, Fr.: Ravensbrück. Edition Le Deporté, Paris 1968.

Aziz, Ph.: I medici dei Lager. Edizioni Ferni, Ginevra 1975.

Bayle, Fr.: Croix gammée contre caducee. Les expériments humaines en Allemagne pendant la deuxième guerre mondiale Selbstverlag, 1950. S.1008-1128; expériences chirurgicales. 1.Sulfonamides; $\mathrm{Os}$, nerfs et muscles.

Bellak, G. Melpdia, G.: Donne e bambini nei Lager nazisti. Aned, Milano 1960.

Bernadac, Ch.: Le camp des femmes, Ravensbrück. ivi, 1972.

- L'holocauste oublié. Le massacre des tsiganes. France - Empire, Paris 1979.

Buber-Neumann, M.: Als Gefangene bei Stalin und Hitler. DVA, Stuttgart 1958.

Buchmann, E.: Frauen im Konzentrationslager. Verlag Das neue Wort, Stuttgart 1946.

Conquest, R.: The Great Terror. MeMillan, London.

De Gaulle, Geneviève: L'Allemagne jugée par Ravensbrück. Les conférences des Ambassadeurs, No 22. Grands Discours Français et Internationaux. Grandes Editions Françaises, Paris 1947. Institut für Zeitgeschichte 82/91132; 57.

Don Zimmet, P.: Les Conditions d'Existence et l'Etat Sanitaire dans les Camps de Concentration de Femmes Déportées en Allemagne. Thèse No 1867, Université de Genève, 1946. 
Dufournier, D.: Ravensbrück. The Woman's Camp of Death. George Allen et Unwin Ltd., London 1948.

Elling, H.: Frauen im deutschen Widerstand 1933-45. Röderberg, Frankfurt am Main 1978.

Finkelmeier, C.: Die braune Apokalypse.Thüringer Volksverlag, Weimar 1947.

Guérin, A.: La Résistance. Chronique illustré 1930-1950. Livre Club Diderot, Paris 1973.

Herbermann, N.: Der gesegnete Abgrund. Schutzhäftling Nr.6582 im Frauenkonzentrationslager Ravensbrïck. Glock und Lutz, Nürnberg 1968.

Mitscherlich, A./Mielke, F.: Das Diktat der Menschenverachtung. Der Nürnberger Ärzteprozeß und seine Quellen. Schneider, Heidelberg 1947.

- - Medizin ohne Menschlichkeit. Dokumente des Nürnberger Ärzteprozesses. Fischer, Frankfurt am Main 1949.

Müller, Ch.: Die Klempnerkolonne in Ravensbrück. Erinnerungen des Häftlings Nr.10787. Frankfurt am Main 1981.

Rolfi, L.B.: Le donne di Ravensbrück. Testimonianze di deportate politiche italiane. Einaudi, Torino 1978.

Schaeder, H.: Ostern im KZ. Haus und Schule, Berlin 1947.

Schmidt-Fels, L.: Deportiert nach Ravensbrück. Bericht einer Zeugin 1943-1945. Dehnen, Düsseldorf 1981.

ten Boom, C./Sherrill, J.E.: Die Zuflucht. 3. Auflage. Brockhaus, Wuppertal 1979.

Tillion, G.: Ravensbrück. Editions du Seuil, Paris 1973.

Vermehren, S.: Reise durch den letzten Akt. Ein Bericht (10.2.44 bis 29.6.45). Christian Wegner, Hamburg 1946.

Wagner, E.: Meine Erlebnisse nach dem 20. Juli 1944. Institut für Zeitgeschichte (Kk 476).

- Allg. Proz. 8. Britische Kriegsverbrecherprozesse (Akten des Judge Advocate General, JAG), KL Ravensbrück:

Verfahren vom 5.12 .1946 bis $3.2 .1947 \quad$ JAG 225

Verfahren vom 14. 4.1946 bis 26.4.1948 JAG 326

Verfahren vom 5. 5.1946 bis 4.6.1948 JAG 335

Verfahren vom 16. 6.1946 bis 29.6.1948 JAG 333

Verfahren vom 2. 7.1946 bis 5.7 .1948 JAG 330

Verfahren vom 2. 7.1946 bis 21.7.1948 JAG 334

Bundesarchiv, Postfach 320, D-54 Koblenz 1.

- Autorenkollektiv: Frauen-Kz Ravensbrück. Deutscher Verlag der Wissenschaften, Berlin (DDR) 1973.

- Comité International de la Croix-Rouge. Documents sur l'activité du Comité International de la Croix-Rouge en faveur des civils détenus dans les camps de concentration en Allemagne (1939-1945). Genève 1946.

- Der Prozeß gegen die Hauptkriegsverbrecher vor dem Internationalen Militärgerichtshof Nürnberg, 14. November 1945 bis 1. Oktober 1946. Band VI, S. 227-257. Nürnberg 1948.

- Dokumente:

- NO-065 (GB-583) Medizinversuche an Kz-Häftlingen 1942-1944.

- NO-871 (II) Pros. Ex.227: Aussagen von Frau Baj Zofia aus Brüssel am 12. Aug. 1946 in Brüssel über die Humanversuche im FKL Ravensbrück.

- Verschärfung der Prügelstrafe. April 1942. Fa 506/12. 
- Frauen-Konzentrationslager Ravensbrück. Geschildert von Ravensbrücker Häftlingen. Sternverlag, Wien.

- Frauen im Konzentrationslager Ravensbrück. Provinzialverwaltung Sachsen. Halle (Saale). Druck 33, 1946.

- KL-Hauptlager Ravensbrück. Angaben über die Außenkommandos. In: Comité International de la Croix-Rouge; Vorläufiges Verzeichnis der Konzentrationslager und deren Außenkommandos sowie anderer Haftstätten unter dem Reichsführer SS in Deutschland und deutsch besetzten Gebieten (1933-1945). Arolsen, 1969.

- Les Françaises à Ravensbrück, par l'Amicale de Ravensbrück et l'Associacion des Déportées et Internées de la Résistance. Gallimard, Paris 1965.

- Résistance à Ravensbrück; Session internationale. Jaszowiec, Warszawa 1973.

- SS im Einsatz: Eine Dokumentation über Verbrechen der SS. Kongreß-Verlag, Berlin 1975. Herausgegeben vom Komitee der Antifaschistischen Widerstandskämpfer in der Deutschen Demokratischen Republik, S.221-226.

- Sterblichkeitsstatistik des Amtes D III des WVHA für den Monat August 1943. Nürnberger Dokumente NO-1010.

- Studien zur Geschichte der Konzentrationslager. Schriftenreihe der Vierteljahreshefte für Zeitgeschichte, Nummer 21. Deutsche Verlags-Anstalt, Stuttgart 1970.

- Trials of War Criminals before the Nuernberg Military Tribunals, Volume I,II: "The Medical Case", Nuernberg October 1946 - April 1947. US Government Printing Office, Washington D.C.

- Über menschliches Maß. Opfer der Hölle Ravensbrück sprechen. Interpress-Verlag, Warschau 1970.

- Unterlagen über die 6 Ravensbrücker Prozesse im Public Record Office, Ruskin Avenue, Kew, Richmond Surrey, TW94DU, England.

\section{Summary}

Under evaluation of the often sparse existing reports and documents, the medical aspect of the camp and the situation of the imprisoned women is being described. For the single prisoner the concentration camp represented an extremely great physical and emotional stress which by dificiences of all kinds and infectious diseases led to physical breakdown and to death. The overcrowding of the camp was - from a medical and hygienic point of view-a catastrophe.

The remembrance of the existence of such camps should in our time be a reminder of human dignity and humanity.

Prof. Dr. med. Dr. phil. Hubert Fischer

Hedwigstrasse 4/II

D-8000 München 19 